\section{SILICON PHOTONICS}

\section{Low-loss and compact}

Opt. Express 23, 21334-21342 (2015)

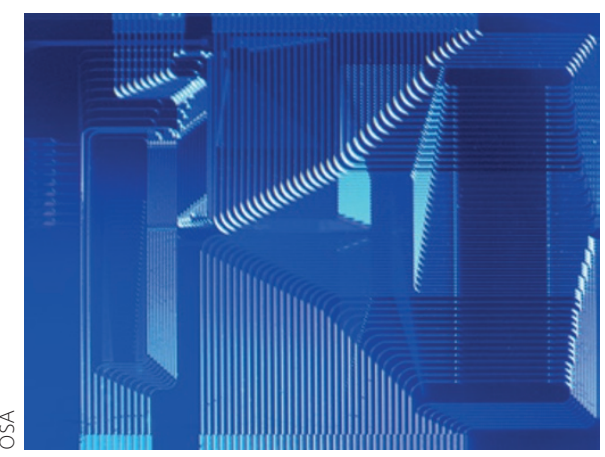

Silicon nitride $\left(\mathrm{Si}_{3} \mathrm{~N}_{4}\right)$ has become a popular material for waveguides in photonic integrated circuits (PICs) because of its low optical propagation losses. Research is now investigating how to implement multilayer, $3 \mathrm{D}$ PICs based on $\mathrm{Si}_{3} \mathrm{~N}_{4}$. To be practical, a $\mathrm{Si}_{3} \mathrm{~N}_{4}$-based 3D optical chip should have low interlayer vertical coupling loss and low waveguide crossing loss. However, there is a trade-off between such losses - a larger gap reduces crossing loss whereas a smaller gap improves vertical transmission. Now, Kuanping Shang and colleagues from University of California, Davis in the USA report a design to help address this problem based on waveguides with a 200 -nm-thick $\mathrm{Si}_{3} \mathrm{~N}_{4}$ core and a $700 \mathrm{~nm}$ interlayer gap. The designed 3D platform offers $0.01 \mathrm{~dB}$ coupling loss from a proposed vertical coupler and multilayer crossing loss of $0.167 \mathrm{~dB}$ at a crossing angle of $90^{\circ}$. A small interlayer crosstalk of only $-52 \mathrm{~dB}$ is measured. The researchers further demonstrate a dual-layer $27 \times 32$ port multilayer star coupler composed of multiple connected couplers, which is useful for 3D PICs. They say that their platform is compatible with active thermal tuning and can be used to make compact photonic devices such as arrayed waveguide gratings.

$R W$

\section{X-RAY PHOTONICS}

\section{Plasma X-ray source}

Phys. Rev. Lett. 115, 083901 (2015)

Soft X-rays have long been an important tool for investigating the properties of material and biological systems and can reveal information about their electronic and magnetic characteristics. To conduct such research, coherent circularly polarized soft X-rays are often desired. However, the generation of circularly polarized soft X-rays is typically limited to a handful of large facilities. There are also emerging laboratory-scale techniques, in particular related to high-harmonic generation, but these sometimes exhibit low photon yields, making single-shot experiments troublesome. Now, Adrien Depresseux and colleagues from France, Czech Republic and Korea have experimentally demonstrated a $32.8 \mathrm{~nm}$ wavelength, plasma-based soft $\mathrm{X}$-ray laser offering circular polarization. The laser-driven source outputs a Gaussian beam profile with $10^{10}$ photons per pulse, which the team notes should be practical for laboratory experimentation. To achieve this, a $1.36 \mathrm{~J}, 30 \mathrm{fs}$ laser pulse pumps a krypton gas cell with an intensity of $\sim 10^{18} \mathrm{~W} \mathrm{~cm}^{-2}$ at a focal point, creating a 5-mm-long column of ionized plasma. A second laser beam, with an energy of $16 \mathrm{~mJ}$ per $350 \mathrm{fs}$ pulse is optimized to generate a $32.8 \mathrm{~nm}$ harmonic. The divergence of the beam is about $1 \mathrm{mrad}$ and the pulses are a few picoseconds in duration. The polarization of the high harmonic is converted from linear to circular using a fourreflector polarizer set-up with an efficiency of $1.5 \%$ at $32.8 \mathrm{~nm}$.

\section{OPTOMECHANICS}

\section{The sound of topology}

Topological states, also known as chiral edge states, are special robust states of matter that are protected against scatter due to the engineering of a material's band structure. Such states have been observed for electrons, atoms and photons but not yet for phonons in a solid-state material. Now Florian Marquardt's group in Erlangen, Germany report that cavity optomechanics in a photonic crystal may provide a means of implementation. In particular, they demonstrate that the topological properties of sound waves (phonons) in such a material can be tuned by adjusting the amplitude and frequency of a driving laser to control the optomechanical interaction between light and sound. Theoretical analysis suggests that the approach should yield chiral, topologically protected phonon states. The research team emphasizes that no time-dependent modulation of the laser is required and that a single laser field with a suitable phase pattern is sufficient to create the phonon edge states. They also comment that by sweeping the laser frequency a regime is entered where it is no longer possible to view phonons and photons as separate due to the existence of a hybrid band structure.

\section{THZ PHOTODETECTORS

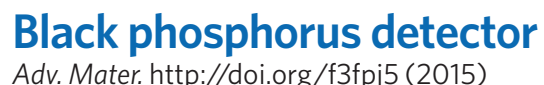 \\ Adv. Mater. http://doi.org/ł3fpj5 (2015)}

Black phosphorus (BP) - a two-dimensional material where the atoms are linked together in puckered sheets - is a promising material for making terahertz $(\mathrm{THz})$ wave detectors due to its high hole mobility exceeding $650 \mathrm{~cm}^{2} \mathrm{~V}^{-1} \mathrm{~s}^{-1}$ and a very high on-off ratio of photo-induced current. Exploiting these advantages, Leonardo Viti and co-workers from Italy, USA, France and Poland have now demonstrated efficient room temperature $\mathrm{THz}$ photodetectors based on a FET that features integrated $\mathrm{THz}$ asymmetric antennas designed to enhance the sensitivity. Flakes of $\mathrm{BP}$ with thicknesses around $10 \mathrm{~nm}$ were mechanically exfoliated from BP crystals grown by chemical vapour transport. The FET device with a resonance frequency of $0.3 \mathrm{THz}$ was fabricated by electron beam lithography and metal evaporation. $\mathrm{THz}$ waves in the frequency range of $0.26-0.38 \mathrm{THz}$ were focused to the FET devices, which exhibited a maximum responsivity and noise-equivalent power of $0.15 \mathrm{~V} \mathrm{~W}^{-1}$ and $40 \mathrm{nW} \mathrm{Hz}^{-1 / 2}$, respectively.

\section{VISION}

\section{Telescopic contact lens Appl. Opt. 54, 7195-7204 (2015)}

Age-related macular degradation (AMD) is a common eye complaint in the elderly, which degrades vision and is the leading cause of blindness for those over the age of 55. Some AMD patients rely on lowmagnification telescopic visual aids to help with the problem. However, such aids are typically bulky and inconvenient. Now, Ashkan Arianpour and co-workers from the USA and Switzerland have developed a 1.6-mm-thick telescopic contact lens that provides both $\times 1$ and $\times 2.8$ magnified vision. The optic features an unmagnified vision path through the central aperture of the lens. This is supplemented with a second vision path based on an 8.2-mm-diameter ring-shaped entrance pupil and four internal reflections in a polymethyl methacrylate precision optic. The design features air gaps to ensure sufficient oxygen flow to the cornea. The switching of the magnifications was implemented by changing the polarization of incoming light. The resolutions of the telescopic lens for each magnification were experimentally tested by using an optomechanical fluid-filled eye model with a full-field view of 34 degrees.

Written by Oliver Graydon, Noriaki Horiuchi, David Pile and Rachel Won. 